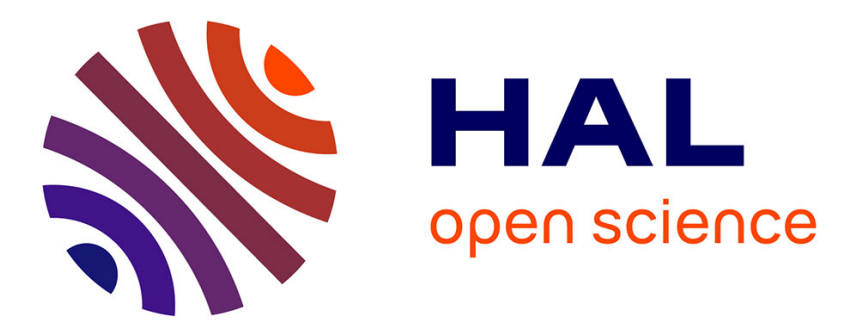

\title{
Mathematical study of non-ideal electrostatic correlations in equilibrium electrolytes
}

\author{
Alexandre Ern, Remi Joubaud, Tony Lelièvre
}

\section{To cite this version:}

Alexandre Ern, Remi Joubaud, Tony Lelièvre. Mathematical study of non-ideal electrostatic correlations in equilibrium electrolytes. 2011. hal-00654736

\section{HAL Id: hal-00654736 \\ https://hal.science/hal-00654736}

Submitted on 22 Dec 2011

HAL is a multi-disciplinary open access archive for the deposit and dissemination of scientific research documents, whether they are published or not. The documents may come from teaching and research institutions in France or abroad, or from public or private research centers.
L'archive ouverte pluridisciplinaire HAL, est destinée au dépôt et à la diffusion de documents scientifiques de niveau recherche, publiés ou non, émanant des établissements d'enseignement et de recherche français ou étrangers, des laboratoires publics ou privés. 


\title{
Mathematical study of non-ideal electrostatic correlations in equilibrium electrolytes
}

\author{
Alexandre Ern ${ }^{1}$, Rémi Joubaud ${ }^{1,2}$ and Tony Lelièvre ${ }^{1,3}$ \\ ${ }^{1}$ Université Paris-Est, CERMICS, École des Ponts, 6 et 8 avenue Blaise Pascal, \\ 77455 Marne-La-Vallée cedex 2, France \\ 2 ANDRA, DRD/EAP, Parc de la croix blanche, 1,7 rue Jean Monnet, 92298 \\ Châtenay-Malabry cedex, France \\ 3 INRIA Rocquencourt, MICMAC Team-Project, Domaine de Voluceau, B.P. 105, \\ 78153 Le Chesnay cedex, France \\ E-mail: ern@cermics.enpc.fr,joubaudr@cermics.enpc.fr, \\ lelievre@cermics.enpc.fr
}

\begin{abstract}
We undertake the mathematical analysis of a model describing equilibrium binary electrolytes surrounded by charged solid walls. The problem is formulated in terms of the electrostatic potential and the ionic concentrations which have prescribed spatial mean values. The free energy of the system is decomposed as the difference of the internal energy and entropy functionals. The entropy functional is the sum of an ideal entropy and an excess entropy, the latter taking into account nonideality due to electrostatic correlations at low ionic concentrations and steric exclusion effects at high ionic concentrations. We derive sufficient conditions to achieve convexity of the entropy functional, yielding a convex-concave free energy functional. Our main result is the existence and uniqueness of the saddle point of the free energy functional and its characterization as a solution of the original model problem. The proof hinges on positive uniform lower bounds for the ionic concentrations and uniform upper bounds for the ionic concentrations and the electrostatic potential. Some numerical experiments are presented in the case where the excess entropy is evaluated using the Mean Spherical Approximation.
\end{abstract}




\section{Introduction}

The motivation for this work is the underground storage of radioactive waste in clay host rocks, whereby a thorough understanding of the clay behavior is of paramount importance. Clays are complex multiscale media. At the scale of one to several nanometers, they can be viewed as a porous medium in which a negatively charged solid matrix confines an electrolyte consisting of a solvent (water) and dissolved ionic species. Confined electrolytes are encountered in many other fields of application with significant interest, e.g., semiconductor devices and chemical engineering. In this work, we consider equilibrium electrolytes, that is, at steady state and no flow. The properties of such systems constitute the first step to understand more complex chemical and mechanical behaviors. For a recent numerical analysis of the coupling with the flow equations, we refer to Prohl and Schmuck [18].

In continuum models, equilibrium electrolytes can be described by the electrostatic potential and the ionic concentrations. For nanometric confinements, the classical Poisson-Boltzmann theory, where the electrostatic potential $\psi$ solves a Poisson equation while the ionic concentrations $\left(c_{i}\right)_{1 \leq i \leq M}$ of the $M$ species follow the Boltzmann distribution, is not sufficient. A first possibility is to resort to direct molecular simulations (see, e.g, Dufrêche et al. [6]) with suitable interaction potentials, but, at nanometric scales and beyond, this approach entails substantial computational costs. An alternative approach is to enrich the Poisson-Boltzmann theory by a more elaborate microscopic description. In what follows, we focus more specifically on ion-ion interactions. In the so-called primitive model, ions are described by charged hard-spheres of diameter $\left(\sigma_{i}\right)_{1 \leq i \leq M}$, and the correlations between the ions are evaluated using the Ornstein-Zernike integral equation linking the direct and indirect correlation functions (see, e.g, the reference textbook [8]). This allows one to derive the excess free-energy of the equilibrium system. The Ornstein-Zernike integral equation needs a closure relation to be solved. Various approaches can be adopted. Without being exhaustive, we mention the Hyper-Netted Chain approximation (HNC), which is a numerical approach, and the Mean Spherical Approximation (MSA) derived by Lebowitz et al. [13, 14] and Blum [3], which is analytical in some physical cases. The interesting feature of the MSA is that it generalizes the Debye-Hückel theory of electrolytes to the case of nonzero ion diameters. Brownian dynamics simulations (where the solvent is a continuum but the ions are still charged explicit particles) within the primitive model have been performed by Jardat et al. [10] and compared to direct molecular simulations and to continuum models within the MSA.

The goal of the present work is to analyze mathematically equilibrium electrolytes including non-ideal effects due to electrostatics correlations at low to intermediate ionic concentrations and steric exclusion effects at high ionic concentrations. We consider binary electrolytes consisting of a counter-ion (cation) and a co-ion (anion); we denote the respective concentrations by $c:=\left(c_{+}, c_{-}\right)$. Non-ideal effects are described by activity coefficients in the electrochemical potentials. Electrostatic correlations are modelled 
through an activity coefficient $\log \gamma_{0}$ which depends on a so-called screening length which itself can be evaluated in terms of the ionic concentrations. This model includes in particular the case where the MSA is used to evaluate the screening length (see $\$ 2.2$ below for further description). Additionally, we account for a hard-sphere contribution which is modelled here as the lowest order term in the Percus-Yervick approximation, leading to a quadratic term in the ionic concentrations.

The mathematical study of critical points of the free-energy of equilibrium electrolytes has been addressed previously in the literature, either in the PoissonBoltzmann setting or some of its generalizations. In [16], Li analyzes the generalized Poisson-Boltzmann theory with implicit solvent. This formulation, also considered by Borukhov, Andelman, and Orland [4], accounts for steric exclusion effects, but not for electrostatic correlations. For an electrolyte with $M$ species, the solvent concentration $c_{0}$ is introduced such that

$$
\sigma^{3} c_{0}:=1-\sum_{i=1}^{M} \sigma^{3} c_{i},
$$

where $\sigma>0$ represents the mean ionic diameter, and the ideal contribution of the solvent concentration, $c_{0}\left(\log \left(\sigma^{3} c_{0}\right)-1\right)$, is included in the entropy functional. The mathematical analysis has been extended to different ionic diameters by $\mathrm{Li}$ [15]. The critical points of the free-energy are sought in a convex set enforcing $c_{i} \geq 0$ for all $0 \leq i \leq M$, so that the ionic concentrations are a priori bounded from above. One important result in the analysis of $[15,16]$ is the proof that these constraints are not active, that is, that all the $c_{i}$ 's, $0 \leq i \leq M$, are bounded uniformly away from zero. The technique of proof, which consists in further optimizing the free-energy by modifying the ionic concentrations at extreme values if the above abounds are not satisfied, will be adapted here to the present context. This extension is not straightforward owing to the coupling of all ionic concentrations in the non-ideal terms. Furthermore, the work of Carlen et al. [5] addresses a similar model using the Green operator for the Poisson equation. The analysis is concerned with nonlinearities of the same kind as those described by the implicit solvent. The mathematical analysis shows that $L^{\infty}$-bounds on the concentrations can be enforced in the convex set where the minimization is taken, but also shows that under a doubling condition on both the ideal and steric exclusion terms, $L^{\infty}$-bounds can be achieved [5, Theorem B.1]. Moreover, the work of Looker treats the case of the Poisson-Boltzmann free-energy in the $\psi$-formulation [17]. The same model is considered by Allaire, Mikelić, and Piatniski [2] in view of homogenization of confined electrolytes coupled with Stokes flow.

Our main result, Theorem 1 below, states that there is a unique saddle point $(\psi, c)$ of the free-energy for an equilibrium binary electrolyte confined by charged walls. The electrostatic potential $\psi$ is sought in the Sobolev space $H^{1}$ with zero mean-value, while the ionic concentrations $c=\left(c_{+}, c_{-}\right)$are sought in the closed convex subset of $L^{2} \times L^{2}$ consisting of nonnegative ionic concentrations with prescribed meanvalue. Moreover we prove that $\psi$ and $c_{ \pm}$are in $L^{\infty}(\Omega)$, and that $c_{ \pm}$are uniformly 
bounded away from zero. Theorem 1 is established under four main assumptions stated in $§ 3.1$. The first two assumptions are the classical bulk electro-neutrality condition on the prescribed mean ionic concentrations and elliptic regularity for the Poisson problem governing the electrostatic potential (with non-homogeneous Neumann boundary conditions). The other two conditions are formulated in an abstract setting for the activity coefficient $\log \gamma_{0}$ describing non-ideal electrostatic correlations. These conditions, which in particular encompass the MSA setting, require a sublinear growth condition at large ionic concentrations for $\log \gamma_{0}$, and a lower bound on the derivative of $\log \gamma_{0}$ with respect to the ionic strength. This last condition is important to assert the convexity of the entropy functional. Indeed, the ideal entropy and the hard-sphere contribution, which dominate respectively at very low and large ionic concentrations, are both convex, but the electrostatic correlations lead to a nonconvex contribution to the entropy functional. Note that we do not need to enforce a priori $L^{\infty}$-bounds on the concentrations. To the best of our knowledge, the present analysis, together with the ongoing work [1] investigating the role of non-ideality for homogenized ion transport in porous media, is the first to address mathematically the critical points of the free-energy in the presence of electrostatic correlations.

This work is organized as follows. In $\S 2$, we give some details on the electrochemical model, including the modelling of non-ideality, the non-dimensionalization of the governing equations, and the free-energy and entropy functionals. Then, in $\S 3$, we state the mathematical assumptions together with our main result, and we present the main steps of its proof. We devote $\S 4$ to the proof of various technical lemmas, dealing in particular with the convexity of the entropy functional and the a priori bounds on the electrostatic potential and ionic concentrations. Finally, some numerical illustrations are presented in $\S 5$; a more detailed discussion of the electrochemical aspects of these results will be reported elsewhere.

\section{Electrochemical model}

\subsection{Geometry and conservation equations}

We consider a periodic setting with elementary cell $\left[0, L_{*}\right]^{d}, d \in\{2,3\}$, with length scale $L_{*}$ (expressed in $\mathrm{m}$ ). The elementary cell contains inclusions $\Omega_{S}$ whose boundary $\partial \Omega_{S}$ contains negative charges with surface density $\Sigma_{S}$ (expressed in $\mathrm{Cm}^{-2}$ ) (see Figure 1 left). Our approach also applies to other settings, e.g., confined electrolytes in nanochannels (see Figure 1 right). The problem is posed in the domain $\Omega:=\left[0, L_{*}\right]^{d} \backslash \Omega_{S}$ and consists in finding the electrostatic potential $\psi$ (expressed in $\mathrm{V}$ ) and the ionic concentrations $c=\left(c_{+}, c_{-}\right)$(each concentration is expressed in $\mathrm{m}^{-3}$ ) such that

$$
\begin{aligned}
& -\Delta \psi=\frac{\mathrm{e}}{\varepsilon} \sum_{i= \pm} Z_{i} c_{i} \quad \text { in } \Omega \\
& \mu_{+}(\psi, c) \text { and } \mu_{-}(\psi, c) \text { are constant in } \Omega
\end{aligned}
$$



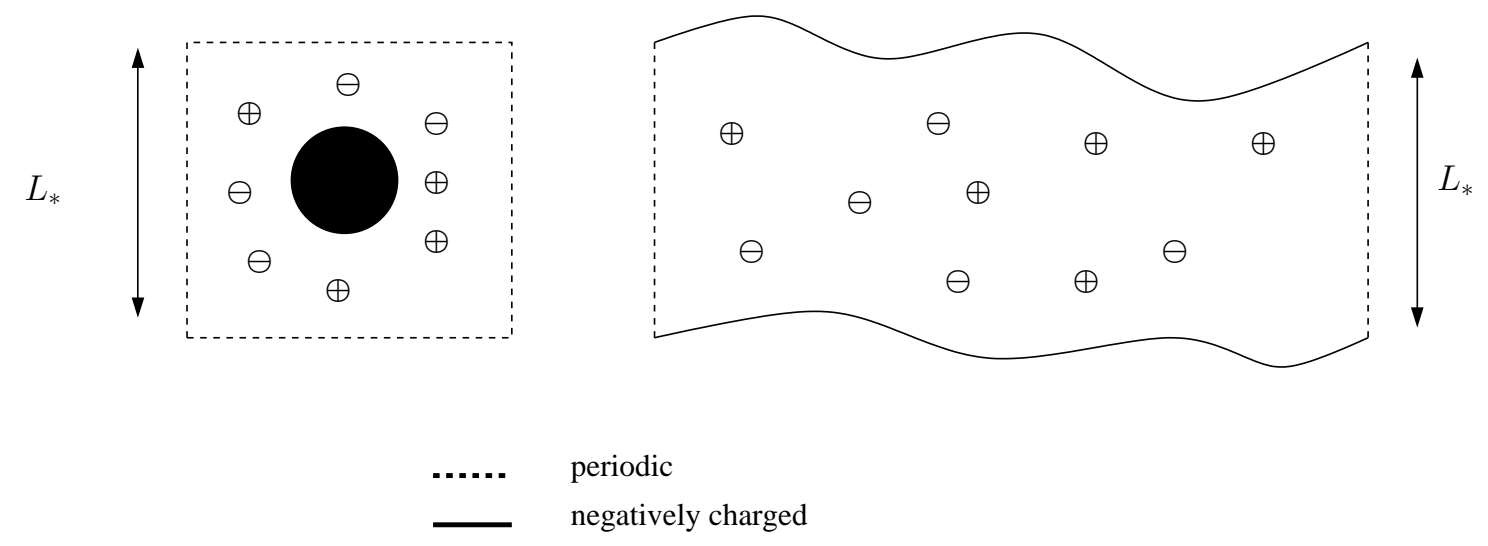

Figure 1. Geometries for $\Omega$ : periodic media with negatively charged inclusions (left); nanochannel with negatively charged walls (right)

where $\mathrm{e}$ is the elementary charge (expressed in $\mathrm{C}$ ), $\varepsilon=\varepsilon_{0} \varepsilon_{r}$ the solvent permittivity with $\varepsilon_{0}$ the vacuum permittivity (expressed in $\mathrm{CV}^{-1} \mathrm{~m}^{-1}$ ) and $\varepsilon_{r}$ the solvent relative permittivity (dimensionless), $Z_{i}$ the valence (dimensionless) of species $i= \pm$, while the electrochemical potentials $\mu_{ \pm}(\psi, c)$ are given by

$$
\mu_{i}(\psi, c)=k_{\mathrm{B}} T \log \left(\sigma^{3} c_{i}\right)+k_{\mathrm{B}} T \log \left(\gamma_{i}(c)\right)+Z_{i} e \psi, \quad i= \pm,
$$

where $k_{\mathrm{B}}$ is the Boltzmann constant (expressed in $\mathrm{JK}^{-1}$ ), $T$ the temperature (expressed in $\mathrm{K}$ ), $\sigma$ the mean ionic diameter (expressed in $\mathrm{m}$ ), and $\gamma_{i}(c)$ the activity coefficient (dimensionless) of species $i$ accounting for non-ideal behavior $\left(\gamma_{i}(c) \equiv 1, i= \pm\right.$, in the ideal case). Boundary conditions enforce that

$$
\begin{aligned}
& \psi \text { is periodic on } \partial \Omega \backslash \partial \Omega_{S}, \\
& \nabla \psi \cdot \boldsymbol{n}=-\frac{1}{\varepsilon} \Sigma_{S} \quad \text { on } \partial \Omega_{S},
\end{aligned}
$$

where $\boldsymbol{n}$ denotes the unit outward normal to $\partial \Omega$. Additionally, we prescribe the mean ionic concentrations in the form

$$
\left\langle c_{i}\right\rangle_{\Omega}=c_{i}^{0}, \quad i= \pm,
$$

where $c_{+}^{0}$ and $c_{-}^{0}$ are given positive real numbers and where, for any function $f \in L^{1}(\Omega)$,

$$
\langle f\rangle_{\Omega}:=\frac{1}{|\Omega|} \int_{\Omega} f
$$

We assume that $c_{ \pm}^{0}$ satisfy the bulk electro-neutrality condition

$$
\sum_{i= \pm} Z_{i} c_{i}^{0}=\frac{1}{|\Omega|} \int_{\partial \Omega} \frac{1}{\mathrm{e}} \Sigma_{S},
$$

which is a necessary and sufficient condition for the solvability of the Poisson equation (1a) together with the boundary conditions (3) for the electrostatic potential $\psi$ given the ionic concentrations $c$. Finally, since the electrostatic potential is determined up to an additive constant, we require that

$$
\langle\psi\rangle_{\Omega}=0 .
$$




\subsection{Modelling of non-ideality}

For each species $i= \pm$, its activity coefficient $\gamma_{i}(c)$, which depends on both ionic concentrations $c_{ \pm}$, is split into two parts in such a way that

$$
\log \left(\gamma_{i}(c)\right)=\log \left(\gamma_{i}^{\mathrm{Coul}}(c)\right)+\log \left(\gamma^{\mathrm{HS}}(c)\right), \quad i= \pm
$$

The first contribution accounts for Coulomb interactions, while the second is a hardsphere term introducing steric effects which dominate at high ionic concentrations.

For the Coulomb term, we consider the mean spherical approximation (MSA) hinging on the screening parameter $\Gamma_{\mathrm{MSA}}$ (expressed in $\mathrm{m}^{-1}$ ) such that

$$
\Gamma_{\mathrm{MSA}}^{2}=\pi L_{\mathrm{B}} \sum_{i= \pm} \frac{Z_{i}^{2} c_{i}}{\left(1+\sigma_{i} \Gamma_{\mathrm{MSA}}\right)^{2}},
$$

where $L_{\mathrm{B}}$ is the Bjerrum length defined by $L_{\mathrm{B}}:=\frac{e^{2}}{4 \pi \varepsilon k_{\mathrm{B}} T}$. Recalling that, in the present work, we consider the mean ion diameter approximation $\sigma_{ \pm}=\sigma$, equation (8) can be solved explicitly. For convenience, we introduce the ionic strength function $I: \mathbb{R}_{\geq 0}^{2} \rightarrow \mathbb{R}$ (expressed in $\mathrm{m}^{-3}$ ) such that, for all $c \in \mathbb{R}_{\geq 0}^{2}$,

$$
I(c):=\sum_{i= \pm} \eta_{i} c_{i}, \quad \eta_{i}:=\frac{1}{2} Z_{i}^{2} .
$$

Then, the screening parameter $\Gamma_{\mathrm{MSA}}$ solving (8) is given by $\Gamma_{\mathrm{MSA}}=\Upsilon_{\mathrm{MSA}}(I(c))$ with the function $\Upsilon_{\mathrm{MSA}}: \mathbb{R}_{\geq 0} \rightarrow \mathbb{R}_{\geq 0}$ such that, for all $\theta \in \mathbb{R}_{\geq 0}$,

$$
\Upsilon_{\mathrm{MSA}}(\theta):=\frac{1}{2 \sigma}\left(\sqrt{2 \sigma\left(4 \pi L_{\mathrm{B}}\right)^{1 / 2}(2 \theta)^{1 / 2}+1}-1\right) .
$$

The activity coefficients $\gamma_{i}^{\mathrm{Coul}}(c), i= \pm$, depend on $c$ only through the ionic strength $I(c)$ and are given by

$$
\log \left(\gamma_{i}^{\mathrm{Coul}}(c)\right)=-Z_{i}^{2} \frac{L_{\mathrm{B}} \Upsilon_{\mathrm{MSA}}(I(c))}{1+\sigma \Upsilon_{\mathrm{MSA}}(I(c))}, \quad i= \pm .
$$

It is convenient to introduce the function $\gamma_{0}: \mathbb{R}_{\geq 0} \rightarrow \mathbb{R}_{\geq 0}$ such that, for all $\theta \in \mathbb{R}_{\geq 0}$,

$$
\log \left(\gamma_{0}(\theta)\right):=-\frac{L_{\mathrm{B}} \Upsilon_{\mathrm{MSA}}(\theta)}{1+\sigma \Upsilon_{\mathrm{MSA}}(\theta)}
$$

so that

$$
\log \left(\gamma_{i}^{\text {Coul }}(c)\right)=Z_{i}^{2} \log \left(\gamma_{0}(I(c))\right), \quad i= \pm .
$$

The hard-sphere contribution stems from the lowest order term in the PercusYervick approximation, that is,

$$
\log \left(\gamma^{\mathrm{HS}}(c)\right):=\frac{4 \pi \sigma^{3}}{3}\left(c_{+}+c_{-}\right)
$$

Such expressions have been derived, e.g., by Waisman and Lebowitz [13, 14] and in the form presented above by Blum [3] who treated the case of asymmetric electrolytes for the unrestricted primitive model. 
Remark 1 (Debye-Hückel limit) For extremely low values of the mean ionic diameter, $\sigma \rightarrow 0$, we recover for $\log \gamma_{0}$ the expression derived in the Debye-Hückel theory, namely $\log \gamma_{0}(\theta)=-\left(2 \pi L_{\mathrm{B}}^{3} \theta\right)^{1 / 2}$.

\subsection{Scaling and non-dimensionalization}

There are three length scales in the problem: the characteristic size of the elementary cell $L_{*}$, the mean ionic diameter $\sigma$, and the Bjerrum length $L_{\mathrm{B}}$. It is convenient to introduce the Debye length $L_{\mathrm{D}}$ and the non-dimensional ratio $\lambda$ such that

$$
L_{\mathrm{D}}:=\sqrt{\frac{L_{*}^{3}}{4 \pi L_{\mathrm{B}}}}, \quad \lambda:=\left(\frac{L_{\mathrm{D}}}{L_{*}}\right)^{2}=\frac{L_{*}}{4 \pi L_{\mathrm{B}}} .
$$

The Debye length represents the scale over which the ions screen out the electric field.

In what follows, we use $L_{*}$ as the characteristic length. Moreover, the reference electrostatic potential is the so-called Zeta potential $\psi_{*}:=k_{\mathrm{B}} T / \mathrm{e}$, the reference electrochemical potential is $\mu_{*}:=k_{\mathrm{B}} T$, the reference surface charge density is $\Sigma_{S *}:=$ $k_{\mathrm{B}} T \varepsilon /\left(\mathrm{e} L_{*}\right)$, and the reference ionic concentration is $c_{*}:=L_{*}^{-3}$. Typical values for these quantities are provided in $\S 5$. With these reference values, the governing equations are recast into non-dimensional form, and to alleviate the notation, we use the same symbols for non-dimensional quantities.

The Poisson problem for the electrostatic potential $\psi$ takes the form

$$
\begin{aligned}
& -\lambda \Delta \psi=\sum_{i= \pm} Z_{i} c_{i} \quad \text { in } \Omega, \\
& \psi \text { is periodic on } \partial \Omega \backslash \partial \Omega_{S}, \\
& \nabla \psi \cdot \boldsymbol{n}=-\Sigma_{S} \quad \text { on } \partial \Omega_{S}, \\
& \langle\psi\rangle_{\Omega}=0 .
\end{aligned}
$$

The mean ionic concentrations $c_{ \pm}^{0}$ satisfy the bulk electro-neutrality condition

$$
\sum_{i= \pm} Z_{i} c_{i}^{0}=\frac{\lambda}{|\Omega|} \int_{\partial \Omega} \Sigma_{S}
$$

and the electrochemical potentials are such that

$$
\mu_{i}(\psi, c)=\log \left(\sigma^{3} c_{i}\right)+\log \left(\gamma_{i}(c)\right)+Z_{i} \psi, \quad i= \pm .
$$

The activity coefficients $\gamma_{ \pm}(c)$ are decomposed as in (7). The Coulomb term is still given by (13) with the ionic strength evaluated as in (9) and the function $\gamma_{0}: \mathbb{R}_{\geq 0} \rightarrow \mathbb{R}_{\geq 0}$ now such that, for all $\theta \in \mathbb{R}_{\geq 0}$,

$$
\log \left(\gamma_{0}(\theta)\right):=-\frac{1}{4 \pi \lambda} \frac{\Upsilon_{\mathrm{MSA}}(\theta)}{1+\sigma \Upsilon_{\mathrm{MSA}}(\theta)},
$$

with the function $\Upsilon_{\mathrm{MSA}}: \mathbb{R}_{\geq 0} \rightarrow \mathbb{R}_{\geq 0}$ such that, for all $\theta \in \mathbb{R}_{\geq 0}$,

$$
\Upsilon_{\mathrm{MSA}}(\theta):=\frac{1}{2 \sigma}\left(\sqrt{2 \sigma \lambda^{-1 / 2}(2 \theta)^{1 / 2}+1}-1\right) .
$$

Finally, the hard-sphere term is still given by (14). 


\subsection{Entropy and Free-Energy functionals}

We define the ideal entropy $s_{\text {id }}: \mathbb{R}_{\geq 0} \rightarrow \mathbb{R}$ such that, for all $u \in \mathbb{R}_{\geq 0}$,

$$
s_{\mathrm{id}}(u):= \begin{cases}u\left(\log \left(\sigma^{3} u\right)-1\right), & u>0 \\ 0, & u=0\end{cases}
$$

and the excess entropy $s_{\mathrm{ex}}: \mathbb{R}_{\geq 0}^{2} \rightarrow \mathbb{R}$ (resulting from non-ideality) such that, for all $c=\left(c_{+}, c_{-}\right) \in \mathbb{R}_{\geq 0}^{2}$,

$$
s_{\mathrm{ex}}(c):=2 \Gamma_{0}(I(c))+\frac{2 \pi \sigma^{3}}{3}\left(c_{+}+c_{-}\right)^{2}
$$

where, for all $\theta \in \mathbb{R}_{\geq 0}$,

$$
\Gamma_{0}(\theta):=\int_{0}^{\theta} \log \left(\gamma_{0}\left(\theta^{\prime}\right)\right) \mathrm{d} \theta^{\prime}=-\frac{1}{4 \pi \sigma \lambda}\left(\theta-\frac{8 \lambda \sigma}{3}\left(\Upsilon_{\mathrm{MSA}}(\theta)\right)^{3}-2 \lambda\left(\Upsilon_{\mathrm{MSA}}(\theta)\right)^{2}\right)
$$

The ideal entropy is continuous in $\mathbb{R}_{\geq 0}$ and continuously differentiable in $\mathbb{R}_{>0}$, while the excess entropy is continuously differentiable in $\mathbb{R}_{\geq 0}^{2}$ with

$$
\frac{\partial s_{\mathrm{ex}}}{\partial c_{+}}(c)=\log \left(\gamma_{+}(c)\right), \quad \frac{\partial s_{\mathrm{ex}}}{\partial c_{-}}(c)=\log \left(\gamma_{-}(c)\right) .
$$

The entropy functional $\mathcal{S}$ is such that

$$
\mathcal{S}(c)=\mathcal{S}_{\mathrm{id}}(c)+\mathcal{S}_{\mathrm{ex}}(c)
$$

with

$$
\mathcal{S}_{\mathrm{id}}(c):=\sum_{i= \pm} \int_{\Omega} s_{\mathrm{id}}\left(c_{i}\right), \quad \mathcal{S}_{\mathrm{ex}}(c):=\int_{\Omega} s_{\mathrm{ex}}(c) .
$$

Finally, the free-energy functional $\mathcal{E}$ is such that

$$
\mathcal{E}(\psi, c)=\mathcal{U}(\psi)-\mathcal{S}(c)-\mathcal{B}(\psi, c)
$$

where

$$
\begin{aligned}
\mathcal{B}(\psi, c) & =\sum_{i= \pm} \int_{\Omega} Z_{i} c_{i} \psi \\
\mathcal{U}(\psi) & =\frac{\lambda}{2} \int_{\Omega}|\nabla \psi|^{2}+\lambda \int_{\partial \Omega_{S}} \Sigma_{S} \psi
\end{aligned}
$$

To motivate the free-energy functional $\mathcal{E}$, we observe formally that, if $(\psi, c)$ is a saddle point of $\mathcal{E}$ under the constraints $\langle\psi\rangle_{\Omega}=0$ and $\left\langle c_{i}\right\rangle_{\Omega}=c_{i}^{0}, i= \pm$, and if $\mathcal{E}$ is differentiable at $(\psi, c)$, then $(\psi, c)$ is a critical point of $\mathcal{E}$. Differentiating $\mathcal{E}$ with respect to its first argument shows that $\psi$ solves the Poisson problem (16), while differentiating $\mathcal{E}$ with respect to its second argument shows that the electrochemical potentials $\mu_{ \pm}(\psi, c)$ are constant in $\Omega$ with $\mu_{i}(\psi, c)=\mu_{i}^{0}, i= \pm$, where the $\mu_{i}^{0}$ 's are the Lagrange multipliers associated with the constraints on the mean ionic concentrations. The purpose of the next section is to give a precise mathematical setting for this result. 


\section{Mathematical analysis}

In this section, we introduce a mathematical framework and prove that, under the assumptions stated below, the free-energy functional $\mathcal{E}$ admits a unique saddle point $(\psi, c)$. Moreover, we establish some a priori bounds on $(\psi, c)$ and show that $\psi$ solves the Poisson problem (16) while the electrochemical potentials $\mu_{i}(\psi, c), i= \pm$, defined by (18) are constant in $\Omega$. In what follows, we consider an abstract setting for the one-real variable function $\log \gamma_{0}$ which can be chosen arbitrarily provided assumptions $\left(\mathrm{H}_{\gamma}\{1,2\}\right)$ below are satisfied. The case of the MSA where $\log \gamma_{0}$ is defined by (19) is a special case of application, for which the verification of assumptions $\left(\mathrm{H}_{\gamma}\{1,2\}\right)$ is discussed in $\S 5$.

\subsection{Assumptions}

We consider the sets

$$
\begin{aligned}
\mathfrak{H} & :=\left\{\phi \in H_{\mathrm{per}}^{1}(\Omega),\langle\phi\rangle_{\Omega}=0\right\}, \\
\mathfrak{K} & :=\left\{c=\left(c_{+}, c_{-}\right) \in\left[L^{2}(\Omega)\right]^{2}, c_{ \pm} \geq 0 \text { a.e. in } \Omega,\left\langle c_{ \pm}\right\rangle_{\Omega}=c_{ \pm}^{0}\right\},
\end{aligned}
$$

where the functional spaces $H_{\text {per }}^{1}(\Omega)$ and $L^{2}(\Omega)$ are, respectively, the closure of $C_{\text {per }}^{\infty}(\bar{\Omega})$ for the canonical norms $\|\cdot\|_{H^{1}(\Omega)}$ and $\|\cdot\|_{L^{2}(\Omega)}$. It is clear that $\mathfrak{H}$ is a closed subspace of $H_{\text {per }}^{1}(\Omega)$ and that $\mathfrak{K}$ is a closed convex subset of $\left[L^{2}(\Omega)\right]^{2}$.

In what follows, we make the following assumptions:

- $\left(\mathrm{H}_{c^{0}}\right) \Sigma_{S} \in L^{1}\left(\partial \Omega_{S}\right)$, the real numbers $c_{ \pm}^{0}$ are positive and satisfy the bulk electroneutrality condition (17).

- $\left(\mathrm{H}_{\Omega}\right) \Sigma_{S} \in H^{1 / 2}\left(\partial \Omega_{S}\right)$ and the affine operator $L: L^{2}(\Omega) \rightarrow \mathfrak{H}$ such that, for all $f \in L^{2}(\Omega), L(f) \in \mathfrak{H}$ solves $-\Delta L(f)=f-\langle f\rangle_{\Omega}+|\Omega|^{-1} \int_{\partial \Omega_{S}} \Sigma_{S}$ in $\Omega$ with the Neumann boundary condition $\nabla L(f) \cdot \boldsymbol{n}=-\Sigma_{S}$ on $\partial \Omega_{S}$ and $\langle L(f)\rangle_{\Omega}=0$, is bounded from $L^{2}(\Omega)$ to $H^{2}(\Omega)$.

- $\left(\mathrm{H}_{\gamma} 1\right)$ The function $\theta \mapsto \log \left(\gamma_{0}(\theta)\right)$ is continuous on $\mathbb{R}_{\geq 0}$; moreover, there is $\beta \in[0,1)$ and $\left(C_{1}, C_{2}\right) \in \mathbb{R}_{\geq 0}^{2}$ such that

$$
\forall \theta \in \mathbb{R}_{\geq 0}, \quad\left|\log \left(\gamma_{0}(\theta)\right)\right| \leq C_{1}+C_{2} \theta^{\beta} .
$$

- $\left(\mathrm{H}_{\gamma} 2\right)$ The function $\theta \mapsto \log \left(\gamma_{0}(\theta)\right)$ is non-increasing and continuously differentiable on $\mathbb{R}_{>0}$ and there holds, for all $\theta \in \mathbb{R}_{>0}$,

$$
\frac{\eta_{\sharp}}{\theta}+\frac{4 \pi \sigma^{3}}{3}+\left(2 \eta_{\sharp}^{2}+\frac{2 \pi \sigma^{3}}{3 \eta_{b}} \theta\left(\eta_{\sharp}-\eta_{b}\right)^{2}\right)\left(\log \gamma_{0}\right)^{\prime}(\theta)>0,
$$

where $\eta_{\sharp}:=\max \left(\eta_{+}, \eta_{-}\right)$and $\eta_{b}:=\min \left(\eta_{+}, \eta_{-}\right)$.

An important consequence of assumption $\left(\mathrm{H}_{\gamma} 1\right)$ is that the excess entropy $s_{\text {ex }}$ is a nondecreasing function of both its arguments if at least one ionic concentration is large enough. 
Lemma 1 Assume $\left(\mathrm{H}_{\gamma} 1\right)$. Then, there is $\kappa_{\gamma} \in \mathbb{R}_{\geq 0}$ such that, for all $c \in \mathbb{R}_{\geq 0}^{2}$ satisfying $c_{+} \geq \kappa_{\gamma}$ or $c_{-} \geq \kappa_{\gamma}$, there holds

$$
\frac{\partial s_{\mathrm{ex}}}{\partial c_{+}}(c) \geq 0, \quad \frac{\partial s_{\mathrm{ex}}}{\partial c_{-}}(c) \geq 0
$$

Proof. Recall that

$$
\frac{\partial s_{\mathrm{ex}}}{\partial c_{ \pm}}(c)=\log \left(\gamma_{ \pm}(c)\right)=2 \eta_{ \pm} \log \left(\gamma_{0}(I(c))+\frac{4 \pi}{3} \sigma^{3}\left(c_{+}+c_{-}\right)\right.
$$

Invoking assumption $\left(\mathrm{H}_{\gamma} 1\right)$, we infer that for suitable constants $C_{3}$ and $C_{4}$, there holds

$$
\frac{\partial s_{\mathrm{ex}}}{\partial c_{ \pm}}(c) \geq \frac{4 \pi}{3} \sigma^{3}\left(c_{+}+c_{-}\right)-C_{3}-C_{4}\left(c_{+}^{\beta}+c_{-}^{\beta}\right) .
$$

Since $\beta \in[0,1)$, the conclusion is straightforward.

\subsection{Main result}

It is readily verified that the functional $\mathcal{E}$ maps $\mathfrak{H} \times \mathfrak{K}$ to $\mathbb{R}$. We say that $(\psi, c) \in \mathfrak{H} \times \mathfrak{K}$ is a saddle point of $\mathcal{E}$ if

$$
\forall \tilde{c} \in \mathfrak{K}, \quad \mathcal{E}(\psi, \tilde{c}) \leq \mathcal{E}(\psi, c) \leq \mathcal{E}(\phi, c), \quad \forall \phi \in \mathfrak{H} .
$$

We can now state the main result of this work.

Theorem 1 Assume $\left(\mathrm{H}_{c^{0}}\right),\left(\mathrm{H}_{\Omega}\right)$, and $\left(\mathrm{H}_{\gamma}\{1,2\}\right)$. Then, the functional $\mathcal{E}$ has a unique saddle point $(\psi, c) \in \mathfrak{H} \times \mathfrak{K}$. Moreover, $\psi \in L^{\infty}(\Omega)$ and there are $0<c_{b} \leq c_{\sharp}<+\infty$ such that, for a.e. $x \in \Omega, c_{b} \leq c_{i}(x) \leq c_{\sharp}, i= \pm$. Finally, $\psi$ solves the Poisson problem (16), and the electrochemical potentials $\mu_{i}(\psi, c), i= \pm$, defined by (18) are constant in $\Omega$.

Proof. The proof is decomposed into several steps.

Existence of a saddle point For any $\bar{c} \in \mathfrak{K}$, the functional $\mathfrak{H} \ni \psi \mapsto \mathcal{E}(\psi, \bar{c}) \in \mathbb{R}$ is strictly convex, continuous, and satisfies, for all $\psi \in \mathfrak{H}$ with $\|\psi\|_{H^{1}(\Omega)} \rightarrow+\infty$, $\mathcal{E}(\psi, \bar{c}) \rightarrow+\infty)$. Furthermore, in Lemma 2 below, we use assumption $\left(\mathrm{H}_{\gamma} 2\right)$ to prove that the functional $\mathcal{S}$ is convex on $\mathfrak{K}$, while in Lemma 3 , we use assumption $\left(\mathrm{H}_{\gamma} 1\right)$ to prove that the functional $\mathcal{S}$ is continuous on $\mathfrak{K}$. Hence, for any $\bar{\psi} \in \mathfrak{H}$, the functional $\mathfrak{K} \ni c \mapsto \mathcal{E}(\bar{\psi}, c) \in \mathbb{R}$ is concave and continuous. The entropy functional also satisfies, for all $c \in \mathfrak{K}$ with $\|c\|_{\left[L^{2}(\Omega)\right]^{2}} \rightarrow+\infty, \mathcal{S}(c) \rightarrow+\infty$ and $\mathcal{E}(\bar{\psi}, c) \rightarrow-\infty$ since, at high concentrations, the hard-sphere contribution to the activity coefficient dominates. As a result, we can apply the Ky Fan-Von Neumann theorem [7, Prop. 2.2, p. 161] to infer the existence of a saddle point $(\psi, c)$ of the functional $\mathcal{E}$. 
Characterization and bound on electrostatic potential Let $(\psi, c) \in \mathfrak{H} \times \mathfrak{K}$ be a saddle point of the functional $\mathcal{E}$. Since $\mathcal{E}$ is differentiable with respect to $\psi$ and since $\mathfrak{H}$ is a vector space, there holds

$$
\left\langle\partial_{\psi} \mathcal{E}(\psi, c), \phi\right\rangle=\lambda \int_{\Omega} \nabla \psi \cdot \nabla \phi+\lambda \int_{\partial \Omega_{S}} \Sigma_{S} \phi-\sum_{i= \pm} \int_{\Omega} Z_{i} c_{i} \phi=0, \quad \forall \phi \in \mathfrak{H} .
$$

This shows that $\psi$ solves the Poisson problem (16). Moreover, recalling the affine operator $L: L^{2}(\Omega) \rightarrow \mathfrak{H}$ introduced in assumption $\left(\mathrm{H}_{\Omega}\right)$ and using the bulk electroneutrality condition (17), we infer

$$
\psi=L\left(\frac{1}{\lambda} \sum_{i= \pm} Z_{i} c_{i}\right) .
$$

As a result, $\psi \in H^{2}(\Omega)$, and owing to the Sobolev embedding theorem, $\psi \in L^{\infty}(\Omega)$.

Characterization and bound on ionic concentrations Using assumption $\left(\mathrm{H}_{\gamma} 1\right)$, we prove in Lemmas 4 and 5 below that there are $0<c_{b} \leq c_{\sharp}<+\infty$ such that, for a.e. $x \in \Omega$, $c_{b} \leq c_{i}(x) \leq c_{\sharp}, i= \pm$. Finally, owing again to the uniform lower bound on the ionic concentrations and using Lemma 3, we infer that the functional $\mathcal{E}$ is Gâteauxdifferentiable at $(\psi, c)$ with respect to $c_{ \pm}$along any direction $v \in C_{\text {per }}^{\infty}(\bar{\Omega})$ with $\langle v\rangle_{\Omega}=0$, and there holds

$$
\left\langle\partial_{c_{ \pm}} \mathcal{E}(\psi, c), v\right\rangle=\int_{\Omega} \mu_{ \pm}(\psi, c) v
$$

where $\mu_{ \pm}(\psi, c)$ are the electrochemical potentials defined by (18). Let now $v \in C_{\text {per }}^{\infty}(\bar{\Omega})$. Since $(\psi, c)$ is a saddle point, there holds $\left\langle\partial_{c_{ \pm}} \mathcal{E}(\psi, c), \tilde{v}\right\rangle=0$ with $\tilde{v}=v-\langle v\rangle_{\Omega}$, whence $\int_{\Omega}\left\{\mu_{ \pm}(\psi, c)-\left\langle\mu_{ \pm}(\psi, c)\right\rangle_{\Omega}\right\} v=0$. By density of $C_{\text {per }}^{\infty}(\bar{\Omega})$ in $L^{2}(\Omega)$, this shows that the electrochemical potentials are constant in $\Omega$.

Uniqueness The functional $\mathcal{E}$ is strictly convex in its first argument. Moreover, owing to the uniform lower bound on the ionic concentrations, entropy is strictly convex in $c$ (see, again, Lemma 2 below). This yields uniqueness of the saddle point.

Remark 2 (Critical points are saddle points) It is readily verified using convexity arguments that if $(\psi, c)$ (with $c$ uniformly bounded from above and below) is a critical point of the free-energy functional $\mathcal{E}$, then $(\psi, c)$ is a saddle point of $\mathcal{E}$. In $\S 5$, we will compute an approximation of the saddle point by solving the Euler-Lagrange equations satisfied by the critical point.

\section{Technical results}

In this section, we state and prove the various lemmas invoked in the proof of our main result, Theorem 1 . Our first result concerns the convexity of the entropy functional $\mathcal{S}$. 
Lemma 2 (Convexity of entropy) Assume $\left(\mathrm{H}_{\gamma} 2\right)$. Then, the functional $\mathcal{S}$ is convex on $\mathfrak{K}$ and strictly convex on the subset $\mathfrak{K}_{>0}:=\left\{c \in \mathfrak{K} ; c_{+}>0\right.$ and $c_{-}>0$ a.e. in $\left.\Omega\right\}$.

Proof. It suffices to show that the entropy $s: \mathbb{R}_{\geq 0}^{2} \rightarrow \mathbb{R}$ such that, for all $c=\left(c_{+}, c_{-}\right) \in \mathbb{R}_{\geq 0}^{2}$,

$$
s(c):=s_{\mathrm{id}}\left(c_{+}\right)+s_{\mathrm{id}}\left(c_{-}\right)+s_{\mathrm{ex}}(c)
$$

is convex on $\mathbb{R}_{\geq 0}^{2}$ and strictly convex on $\mathbb{R}_{>0}^{2}$. Moreover, the convexity of $s$ on $\mathbb{R}_{\geq 0}^{2}$ follows from the strict convexity of $s$ on $\mathbb{R}_{>0}^{2}$ and the continuity of $s$. Hence, it is sufficient to address the strict convexity of $s$ on $\mathbb{R}_{>0}^{2}$. Set $\eta_{\sharp}:=\max \left(\eta_{+}, \eta_{-}\right), \eta_{b}:=\min \left(\eta_{+}, \eta_{-}\right)$, and $\bar{\eta}:=\frac{1}{2}\left(\eta_{+}+\eta_{-}\right)$. The Hessian associated with $s$ is given by

$$
\operatorname{Hess}(s)=\left(\begin{array}{cc}
\frac{1}{c_{+}}+A+B \eta_{+}^{2} & A+B \eta_{+} \eta_{-} \\
A+B \eta_{+} \eta_{-} & \frac{1}{c_{-}}+A+B \eta_{-}^{2}
\end{array}\right),
$$

where $A:=\frac{4 \pi}{3} \sigma^{3}$ and $B:=2\left(\log \gamma_{0}\right)^{\prime}(\theta)$ with $\theta=I(c)$. We verify that the trace and the determinant of $H(s)$ are positive. We obtain

$$
c_{+} c_{-} \operatorname{det} \operatorname{Hess}(s)=1+A\left(c_{+}+c_{-}\right)+\left(\eta_{+}^{2} c_{+}+\eta_{-}^{2} c_{-}+A c_{+} c_{-}\left(\eta_{+}-\eta_{-}\right)^{2}\right) B .
$$

Since $c_{+}+c_{-} \geq \theta / \eta_{\sharp}, \eta_{+}^{2} c_{+}+\eta_{-}^{2} c_{-} \leq \eta_{\sharp} \theta, c_{+} c_{-} \leq \theta^{2} /\left(4 \eta_{-} \eta_{+}\right)$, and $B \leq 0$, we infer that, under condition (32), that is,

$$
\frac{\eta_{\sharp}}{\theta}+A+\left(\eta_{\sharp}^{2}+\frac{A}{4 \eta_{b}} \theta\left(\eta_{\sharp}-\eta_{b}\right)^{2}\right) B>0,
$$

there holds $\operatorname{det} \operatorname{Hess}(s)>0$. Furthermore,

$$
\frac{1}{2} \operatorname{tr} \operatorname{Hess}(s)=\frac{1}{2}\left(\frac{1}{c_{+}}+\frac{1}{c_{-}}\right)+A+\frac{1}{2}\left(\eta_{+}^{2}+\eta_{-}^{2}\right) B .
$$

Since $\left(c_{+}^{-1}+c_{-}^{-1}\right) / 2 \geq \bar{\eta} / \theta$, we infer that, under the condition

$$
\frac{\bar{\eta}}{\theta}+A+\frac{1}{2}\left(\eta_{\sharp}^{2}+\eta_{b}^{2}\right) B>0,
$$

there holds $\operatorname{tr} \operatorname{Hess}(s)>0$. Finally, it is readily verified that, for all $\theta \in \mathbb{R}_{>0}$, $\left(\frac{\eta_{\sharp}}{\theta}+A\right) \frac{1}{2}\left(\eta_{+}^{2}+\eta_{-}^{2}\right) \leq\left(\frac{\bar{\eta}}{\theta}+A\right)\left(\eta_{\sharp}^{2}+\frac{A}{4 \eta_{b}} \theta\left(\eta_{\sharp}-\eta_{b}\right)^{2}\right)$, so that (35) implies (36).

Remark 3 (Necessary convexity condition for symmetric electrolytes) In the case of symmetric electrolytes, that is, $\eta_{+}=\eta_{-}=\eta$, condition (32) reduces to

$$
\frac{\eta}{\theta}+\frac{4 \pi \sigma^{3}}{3}+2 \eta^{2}\left(\log \gamma_{0}\right)^{\prime}(\theta)>0
$$

and it is readily verified that this condition is also necessary for the convexity of the entropy. 
Our second result deals with the continuity and Gâteaux-differentiability of the entropy functional $\mathcal{S}$.

Lemma 3 (Continuity and differentiability of entropy) Assume $\left(\mathrm{H}_{\gamma} 1\right)$. Then, the entropy functional $\mathcal{S}$ is continuous on $\left[L^{2}(\Omega)\right]^{2}$. Moreover, for all $c \in\left[L^{2}(\Omega)\right]^{2}$ such that there is $c_{b}>0$ with $c_{i}(x) \geq c_{b}$ for a.e. $x \in \Omega$ and all $i= \pm, \mathcal{S}$ is Gâteauxdifferentiable at $c$ along any direction $v \in C_{\mathrm{per}}^{\infty}(\bar{\Omega})$, and there holds

$$
\left\langle\partial_{c_{ \pm}} \mathcal{S}(c), v\right\rangle=\int_{\Omega}\left\{\log \left(\sigma^{3} c_{ \pm}\right)+\log \left(\gamma_{ \pm}(c)\right)\right\} v .
$$

Proof. A classical result of nonlinear analysis [12, Lemma 16.2, p. 61] states that, if $F: \mathbb{R} \rightarrow \mathbb{R}$ is a continuous function satisfying the growth condition

$$
\exists a, b \in \mathbb{R}, \quad \forall u \in \mathbb{R}, \quad|F(u)| \leq a+b|u|^{p / q},
$$

with $1 \leq p, q<+\infty$ then, for all $v \in L^{p}(\Omega)$, there holds $F(v) \in L^{q}(\Omega)$, and the superposition operator $L^{p}(\Omega) \ni v \mapsto F(v) \in L^{q}(\Omega)$ is continuous. This result can be applied to the function $F_{1}(x)=s_{\text {id }}(x)$ (extended by zero for $x \leq 0$ ) showing that $\mathcal{S}_{\text {id }}(c)$ is continuous from $\left[L^{2}(\Omega)\right]^{2}$ to $L^{1}(\Omega)$. The result can also be applied to the function $F_{2}(x)=\Gamma_{0}(x)$ (extended by zero for $x \leq 0$ ) since, owing to assumption $\left(\mathrm{H}_{\gamma} 1\right), F_{2}$ satisfies the above growth condition with exponent $1+\beta<2$. Since the function $c \mapsto I(c)$ maps continuously $\left[L^{2}(\Omega)\right]^{2}$ to $L^{2}(\Omega)$, we infer that the functional $c \mapsto 2 \Gamma_{0}(I(c))$ is continuous from $\left[L^{2}(\Omega)\right]^{2}$ to $L^{1}(\Omega)$. Finally, the continuity of the hard-sphere contribution $c \mapsto \frac{2 \pi \sigma^{3}}{3}\left(c_{+}+c_{-}\right)^{2}$ from $\left[L^{2}(\Omega)\right]^{2}$ to $L^{1}(\Omega)$ is obvious.

Gâteaux-differentiability Let now $c \in\left[L^{2}(\Omega)\right]^{2}$ such that there is $c_{b}>0$ with $c_{i}(x) \geq c_{b}$ for a.e. $x \in \Omega$ and all $i= \pm$. We treat the partial derivative with respect to $c_{+}$; the other case is treated similarly. Let $v \in C_{\text {per }}^{\infty}(\bar{\Omega})$ with $\langle v\rangle_{\Omega}=0$. There is $t_{0}>0$ such that, for all $t \in\left[-t_{0}, t_{0}\right], c_{+}(x)+t v(x) \geq \frac{1}{2} c_{b}$ for a.e. $x \in \Omega$. Since the function $F_{3}(x)=\log \left(\sigma^{3} x\right)$ for $x \geq \frac{1}{2} c_{b}$ extended by the constant value $\log \left(\frac{1}{2} \sigma^{3} c_{b}\right)$ for $x \leq \frac{1}{2} c_{b}$ satisfies a linear growth condition. Using the proof of [12, Lemma 17.1, p. 64], we can prove that the ideal entropy is Gâteaux-differentiable with

$$
\left\langle\partial_{c_{+}} \mathcal{S}_{\mathrm{id}}(c), v\right\rangle=\int_{\Omega} \log \left(\sigma^{3} c_{+}\right) v
$$

Gâteaux-differentiating the excess entropy is simpler. We use the growth condition in assumption $\left(\mathrm{H}_{\gamma} 1\right)$ to infer

$$
\left\langle\partial_{c_{+}}\left(\int_{\Omega} 2 \Gamma_{0}(I(c))\right), v\right\rangle=\int_{\Omega} 2 \eta_{+} \log \left(\gamma_{0}(I(c)) v=\int_{\Omega} \log \left(\gamma_{+}^{\mathrm{Coul}}(c)\right) v\right.
$$

while Gâteaux-differentiating the hard-sphere term $\frac{2 \pi \sigma^{3}}{3}\left(c_{+}+c_{-}\right)^{2}$ is straightforward. $\diamond$ Our third result delivers an a priori $L^{\infty}$-bound on the ionic concentrations $c=$ $\left(c_{+}, c_{-}\right)$. 
Lemma 4 (Upper bound on $c$ ) Assume $\left(\mathrm{H}_{\gamma} 1\right)$. Let $(\psi, c) \in \mathfrak{H} \times \mathfrak{K}$ be a saddle point of the functional $\mathcal{E}$. Then, there is $c_{\sharp}<+\infty$ such that, for a.e. $x \in \Omega, c_{i}(x) \leq c_{\sharp}$ for all $i= \pm$.

Proof. Let $(\psi, c) \in \mathfrak{H} \times \mathfrak{K}$ be a saddle point of the functional $\mathcal{E}$. Proceeding by contradiction, we assume that there is $i= \pm$ such that, for all $n \in \mathbb{N}$, the set

$$
A_{i}^{n}:=\left\{x \in \Omega ; c_{i}(x)>2^{n}\right\}
$$

has positive measure. We then construct modified ionic concentrations $\tilde{c} \in \mathfrak{K}$ such that $\mathcal{E}(\psi, \tilde{c})>\mathcal{E}(\psi, c)$, thereby providing the desired contradiction with (34). As a result, for all $i= \pm$, there is $n_{i} \in \mathbb{N}$ such that the set $A_{i}^{n_{i}}$ has zero measure, yielding the statement of Lemma 4 with $c_{\sharp}=\max _{i= \pm} 2^{n_{i}}$. The principle of the construction is that, by diminishing the ionic concentration where it is very large, the entropy can be decreased, and thus the free-energy increased. Both the ideal term and the steric exclusion term are large enough at high concentrations to lead to an entropy decrease. We choose to work with the ideal term since, in the proof of Lemma 5 below, the ideal term is the only one leading to the entropy decrease at small concentrations; thus, the two proofs are similar. Furthermore, we observe that some care is needed when perturbing the ionic concentrations since it is necessary to preserve their mean values. Without loss of generality, we assume that, for all $n \in \mathbb{N}$, the set $A_{+}^{n}$ has positive measure. We first observe that there is $k \in \mathbb{N}$ such that the set

$$
\Omega_{+}^{k}:=\left\{x \in \Omega ; 2^{-k} \leq c_{+}(x) \leq 2^{k}\right\}
$$

has positive measure (otherwise, $c_{+}$is zero or infinity a.e. in $\Omega$ which contradicts the fact that $\left\langle c_{+}\right\rangle_{\Omega}=c_{+}^{0}>0$ ). In what follows, we fix such $k \in \mathbb{N}$, and, without loss of generality, we assume that $n \geq k$ so that the sets $A_{+}^{n}$ and $\Omega_{+}^{k}$ are disjoint. Moreover, since $\left\langle c_{-}\right\rangle_{\Omega}=c_{-}^{0}$ and $c_{-}(x) \geq 0$ for a.e. $x \in \Omega$, we infer that, for all $m \in \mathbb{N}, c_{-}^{0} \geq \frac{1}{|\Omega|} 2^{m}\left|A_{-}^{m}\right|$, which shows that $\left|A_{-}^{m}\right| \rightarrow 0$ as $m \rightarrow \infty$. As a result, there is $m \in \mathbb{N}$ such that the set $\Omega^{k, m}:=\Omega_{+}^{k} \cap\left(\Omega \backslash A_{-}^{m}\right)$ has positive measure. In what follows, we fix such $m \in \mathbb{N}$. We observe that in $\Omega^{k, m}$, both ionic concentrations $c_{ \pm}$are bounded by $C_{k, m}:=\max \left(2^{k}, 2^{m}\right)$. We now define the function $\tilde{c}_{+}^{n}$ as follows:

$$
\tilde{c}_{+}^{n}(x)= \begin{cases}0, & x \in A_{+}^{n}, \\ c_{+}(x)+\delta^{n}, & x \in \Omega^{k, m}, \\ c_{+}(x), & x \in \Omega \backslash\left(A_{+}^{n} \cup \Omega^{k, m}\right) .\end{cases}
$$

with $\delta^{n}=\frac{1}{\left|\Omega^{k, m}\right|} \int_{A_{+}^{n}} c_{+}$. It is readily verified that $\left\langle\tilde{c}_{+}^{n}\right\rangle_{\Omega}=c_{+}^{0}$ so that $\tilde{c}^{n}:=\left(\tilde{c}_{+}^{n}, c_{-}\right) \in \mathfrak{K}$. We observe that the real number $\delta^{n}$ is uniformly bounded since $\delta^{n} \leq \delta:=\frac{1}{\left|\Omega^{k, m}\right|}|\Omega| c_{+}^{0}$ for all $n \in \mathbb{N}$. It is important to modify $c_{+}$only in the set $\Omega^{k, m}$ to preserve its mean value (and not in the larger set $\Omega_{+}^{k}$ ); indeed, a bound on $c_{-}$is needed to control the variation of the non-ideal terms between $c$ and $\tilde{c}^{n}$.

To conclude the proof, we show that it is possible to choose $n$ large enough so that

$$
\Delta \mathcal{E}:=\mathcal{E}\left(\psi, \tilde{c}^{n}\right)-\mathcal{E}(\psi, c)>0 .
$$


There holds

$$
\Delta \mathcal{E}=-\Delta \mathcal{S}_{\mathrm{id}}-\Delta \mathcal{S}_{\mathrm{ex}}-\Delta \mathcal{B}
$$

with $\Delta \mathcal{S}_{\text {id }}:=\mathcal{S}_{\text {id }}\left(\tilde{c}^{n}\right)-\mathcal{S}_{\text {id }}(c), \Delta \mathcal{S}_{\text {ex }}:=\mathcal{S}_{\text {ex }}\left(\tilde{c}^{n}\right)-\mathcal{S}_{\text {ex }}(c)$, and $\Delta \mathcal{B}:=\mathcal{B}\left(\psi, \tilde{c}^{n}\right)-\mathcal{B}(\psi, c)$. We estimate the three terms separately. Since

$$
\Delta \mathcal{B}=-Z_{+} \int_{A_{+}^{n}} c_{+} \psi+Z_{+} \int_{\Omega^{k, m}} \delta^{n} \psi
$$

we infer, since $\psi \in L^{\infty}(\Omega)$ owing to the second step in the proof of Theorem 1 , that

$$
|\Delta \mathcal{B}| \leq 2 Z_{+}\|\psi\|_{L^{\infty}(\Omega)} \int_{A_{+}^{n}} c_{+}
$$

Since

$$
\Delta \mathcal{S}_{\mathrm{id}}=\int_{A_{+}^{n}}-s_{\mathrm{id}}\left(c_{+}\right)+\int_{\Omega^{k, m}}\left\{s_{\mathrm{id}}\left(c_{+}+\delta^{n}\right)-s_{\mathrm{id}}\left(c_{+}\right)\right\}=: T_{1}+T_{2},
$$

we infer that

$$
\begin{aligned}
T_{1} & \leq-\left(\log \left(\sigma^{3} 2^{n}\right)-1\right) \int_{A_{+}^{n}} c_{+}, \\
\left|T_{2}\right| & \leq \delta^{n} \int_{\Omega^{k, m}} m\left(c_{+}, c_{+}+\delta^{n}\right) \leq m\left(2^{-k}, 2^{k}+\delta\right) \int_{A_{+}^{n}} c_{+},
\end{aligned}
$$

where we have used the fact that $c_{+} \geq 0, \delta^{n} \leq \delta$, and that, for $b \in \mathbb{R}_{>0}$ and $a \in \mathbb{R}_{\geq 0}$, there holds $\left|s_{\text {id }}(b)-s_{\text {id }}(a)\right| \leq|b-a| m(a, b)$ with $m(a, b):=\max \left(\left|\log \left(\sigma^{3} a\right)\right|,\left|\log \left(\sigma^{3} b\right)\right|\right)$. Turning next to $\Delta \mathcal{S}_{\text {ex }}$, recall that the excess entropy $s_{\text {ex }}$ is continuously differentiable in $\mathbb{R}_{\geq 0}^{2}$ and that $\frac{\partial s_{\mathrm{ex}}}{\partial c_{+}}(u) \geq 0$ for all $u=\left(u_{+}, u_{-}\right) \in \mathbb{R}_{\geq 0}^{2}$ such that $u_{+} \geq \kappa_{\gamma}$ or $u_{-} \geq \kappa_{\gamma}$ as shown in Lemma 1. Let $C_{\gamma}:=\max _{u \in K_{\gamma}}\left|\frac{\partial s_{\mathrm{ex}}}{\partial c_{+}}(u)\right|$ with the compact set $K_{\gamma}:=\left[0, \max \left(2^{k}+\delta, \kappa_{\gamma}\right)\right] \times\left[0, \max \left(2^{m}, \kappa_{\gamma}\right)\right]$. We decompose $\Delta \mathcal{S}_{\text {ex }}$ into

$$
\Delta \mathcal{S}_{\mathrm{ex}}=\int_{A_{+}^{n}}\left\{s_{\mathrm{ex}}\left(\tilde{c}_{+}^{n}, c_{-}\right)-s_{\mathrm{ex}}\left(c_{+}, c_{-}\right)\right\}+\int_{\Omega^{k, m}}\left\{s_{\mathrm{ex}}\left(\tilde{c}_{+}^{n}, c_{-}\right)-s_{\mathrm{ex}}\left(c_{+}, c_{-}\right)\right\}=: T_{3}+T_{4} .
$$

Observing that $s_{\text {ex }}\left(\tilde{c}_{+}^{n}, c_{-}\right)-s_{\text {ex }}\left(c_{+}, c_{-}\right)=\left(\int_{c_{+}}^{\tilde{c}_{+}^{n}} \frac{\partial s_{\mathrm{ex}}}{\partial c_{+}}\left(u_{+}, c_{-}\right) \mathrm{d} u_{+}\right)$, we obtain

$$
\left|T_{4}\right| \leq \int_{\Omega^{k, m}} C_{\gamma} \delta^{n}=C_{\gamma} \int_{A_{+}^{n}} c_{+},
$$

since for all $x \in \Omega^{k, m}$ and for all $u_{+} \in\left[c_{+}(x), \tilde{c}_{+}^{n}(x)\right],\left(u_{+}, c_{-}(x)\right) \in K_{\gamma}$. Moreover, owing to Lemma 1 ,

$$
\begin{aligned}
T_{3} & \leq \int_{A_{+}^{n}}\left\{s_{\mathrm{ex}}\left(0, c_{-}\right)-s_{\mathrm{ex}}\left(\min \left(\kappa_{\gamma}, c_{+}\right), c_{-}\right)\right\} \\
& \leq \int_{A_{+}^{n} \cap\left\{c_{-} \leq \kappa_{\gamma}\right\}}\left\{s_{\mathrm{ex}}\left(0, c_{-}\right)-s_{\mathrm{ex}}\left(\min \left(\kappa_{\gamma}, c_{+}\right), c_{-}\right)\right\}=: T_{3}^{\prime},
\end{aligned}
$$


since for $c_{-}>\kappa_{\gamma}, s_{\mathrm{ex}}\left(0, c_{-}\right)-s_{\mathrm{ex}}\left(\min \left(\kappa_{\gamma}, c_{+}\right), c_{-}\right) \leq 0$. Moreover,

$$
\begin{aligned}
\left|T_{3}^{\prime}\right| & \leq \int_{A_{+}^{n} \cap\left\{c_{-} \leq \kappa_{\gamma}\right\}}\left(\int_{0}^{\min \left(\kappa_{\gamma}, c_{+}\right)}\left|\frac{\partial s_{\mathrm{ex}}}{\partial c_{+}}\left(u_{+}, c_{-}\right)\right| \mathrm{d} u_{+}\right) \\
& \leq \int_{A_{+}^{n} \cap\left\{c_{-} \leq \kappa_{\gamma}\right\}} C_{\gamma} \min \left(\kappa_{\gamma}, c_{+}\right) \leq C_{\gamma} \int_{A_{+}^{n}} c_{+} .
\end{aligned}
$$

Collecting the above bounds, we infer

$$
\Delta \mathcal{E} \geq\left(\log \left(\sigma^{3} 2^{n}\right)-1-C\right) \int_{A_{+}^{n}} c_{+}
$$

with $C=2 Z_{+}\|\psi\|_{L^{\infty}(\Omega)}+m\left(2^{-k}, 2^{k}+\delta\right)+2 C_{\gamma}$. Taking $n$ large enough so that $\log \left(\sigma^{3} 2^{n}\right) \geq 1+C$ and since $A_{+}^{n}$ has positive measure, we infer $\Delta \mathcal{E}>0$.

Our last result delivers a uniform positive lower bound on the ionic concentrations.

Lemma 5 (Uniform positive lower bound on $c$ ) Assume $\left(\mathrm{H}_{\gamma} 1\right)$. Let $(\psi, c) \in \mathfrak{H} \times$ $\mathfrak{K}$ be a saddle point of the functional $\mathcal{E}$. Then, there is $c_{b}>0$ such that, for a.e. $x \in \Omega$, $c_{i}(x) \geq c_{b}$ for all $i= \pm$.

Proof. Let $(\psi, c) \in \mathfrak{H} \times \mathfrak{K}$ be a saddle point of the functional $\mathcal{E}$. The structure of the proof is similar to that of Lemma 4, though a bit simpler since we already have upper bounds on $c_{ \pm}$. Proceeding by contradiction, we assume that there is $i= \pm$ such that, for all $n \in \mathbb{N}$, the set

$$
B_{i}^{n}:=\left\{x \in \Omega ; c_{i}(x)<2^{-n}\right\}
$$

has positive measure. We then construct modified ionic concentrations $\tilde{c} \in \mathfrak{K}$ such that $\mathcal{E}(\psi, \tilde{c})>\mathcal{E}(\psi, c)$, thereby providing the desired contradiction with (34). As a result, for all $i= \pm$, there is $n_{i} \in \mathbb{N}$ such that the set $B_{i}^{n_{i}}$ has zero measure, yielding the statement of Lemma 5 with $c_{b}=\min _{i= \pm} 2^{-n_{i}}$.

Without loss of generality, we assume that, for all $n \in \mathbb{N}$, the set $B_{+}^{n}$ has positive measure. We fix $k \in \mathbb{N}$ such that the set $\Omega_{+}^{k}$ defined by (38) has positive measure, and, without loss of generality, we assume that $n$ is large enough so that $2^{-n} \leq \frac{1}{|\Omega|}\left|\Omega_{+}^{k}\right| 2^{-k}$. Since this implies $2^{-n} \leq 2^{-k}$, the sets $B_{+}^{n}$ and $\Omega_{+}^{k}$ are disjoint. We now define the function $\tilde{c}_{+}^{n}$ as follows:

$$
\tilde{c}_{+}^{n}(x)= \begin{cases}c_{+}(x)+2^{-n}, & x \in B_{+}^{n}, \\ c_{+}(x)-\delta^{n}, & x \in \Omega_{+}^{k}, \\ c_{+}(x), & x \in \Omega \backslash\left(B_{+}^{n} \cup \Omega_{+}^{k}\right) .\end{cases}
$$

with $\delta^{n}=\frac{1}{\left|\Omega_{+}^{k}\right|}\left|B_{+}^{n}\right| 2^{-n}$. It is readily verified that $\left\langle\tilde{c}_{+}^{n}\right\rangle_{\Omega}=c_{+}^{0}$. Moreover, since $\left|B_{+}^{n}\right|<|\Omega|, \delta^{n}<\frac{1}{\left|\Omega_{+}^{k}\right|}|\Omega| 2^{-n} \leq 2^{-k}$, so that $\tilde{c}_{+}^{n} \geq 0$ in $\Omega$. Hence, $\tilde{c}^{n}:=\left(\tilde{c}_{+}^{n}, c_{-}\right) \in \mathfrak{K}$.

To conclude the proof, we show that it is possible to choose $n$ large enough so that

$$
\Delta \mathcal{E}:=\mathcal{E}\left(\psi, \tilde{c}^{n}\right)-\mathcal{E}(\psi, c)>0
$$


As in the proof of Lemma 4 , we write $\Delta \mathcal{E}=-\Delta \mathcal{S}_{\text {id }}-\Delta \mathcal{S}_{\text {ex }}-\Delta \mathcal{B}$. Since $\psi \in L^{\infty}(\Omega)$ owing to the second step in the proof of Theorem 1, we infer

$$
|\Delta \mathcal{B}| \leq 2 Z_{+}\|\psi\|_{L^{\infty}(\Omega)} 2^{-n}\left|B_{+}^{n}\right|
$$

Moreover,

$$
\Delta \mathcal{S}_{\mathrm{id}}=\int_{B_{+}^{n}}\left\{s_{\mathrm{id}}\left(c_{+}+2^{-n}\right)-s_{\mathrm{id}}\left(c_{+}\right)\right\}+\int_{\Omega_{+}^{k}}\left\{s_{\mathrm{id}}\left(c_{+}-\delta^{n}\right)-s_{\mathrm{id}}\left(c_{+}\right)\right\}=: T_{1}+T_{2},
$$

with

$$
\begin{gathered}
T_{1} \leq \int_{B_{+}^{n}} 2^{-n} \log \left(\sigma^{3} 2^{-n+1}\right)=\log \left(\sigma^{3} 2^{-n+1}\right) 2^{-n}\left|B_{+}^{n}\right| \\
\left|T_{2}\right| \leq \int_{\Omega_{+}^{k}} \delta^{n} m\left(c_{+}-\delta^{n}, c_{+}\right) \leq m\left(2^{-k}-\delta, 2^{k}\right) 2^{-n}\left|B_{+}^{n}\right| .
\end{gathered}
$$

Finally, let $C_{\gamma}^{\prime}:=\max _{u \in K_{\gamma}^{\prime}}\left|\frac{\partial s_{\mathrm{ex}}}{\partial c_{+}}(u)\right|$ with the compact set $K_{\gamma}^{\prime}:=\left[0,2^{k}\right] \times\left[0, c_{\sharp}\right]$. We obtain

$$
\left|\Delta \mathcal{S}_{\mathrm{ex}}\right| \leq \int_{B_{+}^{n} \cup \Omega_{+}^{k}}\left(\int_{c_{+}}^{\tilde{c}_{+}^{n}}\left|\frac{\partial s_{\mathrm{ex}}}{\partial c_{+}}\left(u_{+}, c_{-}\right)\right| \mathrm{d} u_{+}\right) \leq C_{\gamma}^{\prime} \int_{B_{+}^{n} \cup \Omega_{+}^{k}}\left|\tilde{c}_{+}^{n}-c_{+}\right| \leq 2 C_{\gamma}^{\prime} 2^{-n}\left|B_{+}^{n}\right|,
$$

since for all $x \in B_{+}^{n} \cup \Omega_{+}^{k}$ and for all $u_{+} \in\left[c_{+}(x), \tilde{c}_{+}^{n}(x)\right]\left(u_{+}, c_{-}(x)\right) \in K_{\gamma}^{\prime}$. Collecting the above bounds, we infer

$$
\Delta \mathcal{E} \geq\left(\log \left(\sigma^{-3} 2^{n-1}\right)-C\right) 2^{-n}\left|B_{+}^{n}\right|
$$

with $C=2 Z_{+}\|\psi\|_{L^{\infty}(\Omega)}+m\left(2^{-k}-\delta, 2^{k}\right)+2 C_{\gamma}^{\prime}$. Taking $n$ large enough so that $\log \left(\sigma^{-3} 2^{n-1}\right) \geq C$ and since $B_{+}^{n}$ has positive measure, we infer $\Delta \mathcal{E}>0$.

\section{Numerical illustration}

This section presents a numerical experiment in the case where the activity coefficient $\log \gamma_{0}$ is evaluated using the MSA.

\subsection{Verifications of assumptions for $M S A$}

We verify the abstract assumptions $\left(\mathrm{H}_{\gamma}\{1,2\}\right)$ in the context of the MSA, that is, when $\log \gamma_{0}$ is defined by (19) with the screening parameter $\Upsilon_{\mathrm{MSA}}$ defined by (20). Assumption $\left(\mathrm{H}_{\gamma} 1\right)$ is straightforward to verify, so that we focus on $\left(\mathrm{H}_{\gamma} 2\right)$. For all $\theta>0$, setting $y:=\sqrt{2 \sigma \lambda^{-1 / 2}(2 \theta)^{1 / 2}+1}$, we obtain

$$
\left(\log \gamma_{0}\right)^{\prime}(\theta)=-\frac{\sigma}{\pi \lambda^{2}} \frac{1}{y(y+1)^{2}\left(y^{2}-1\right)},
$$


and a simple calculation shows that $\left(\mathrm{H}_{\gamma} 2\right)$ is equivalent to the fact that the sixth degree polynomial

$$
\begin{aligned}
P(y)= & y(y-1)^{2}(y+1)^{3}-\frac{\left(\eta_{\sharp}-\eta_{b}\right)^{2}}{16 \pi \lambda \sigma \eta_{b}}\left(y^{2}-1\right)^{2}(y-1) \\
& +\left(\frac{6 \eta_{\sharp}}{\pi \lambda \sigma}\right) y(y+1)-\frac{3}{2}\left(\frac{\eta_{\sharp}}{\pi \lambda \sigma}\right)^{2}(y-1)
\end{aligned}
$$

takes positive values for all $y>1$. This condition, in turn, can be checked numerically. It holds true under a condition of the form $\sigma \lambda>v_{0}$, where the threshold $v_{0}$, which depends on the species valences $Z_{ \pm}$, is reported in the second column of Table 1 for several values of $Z_{ \pm}$. Recalling the definition (15) of the non-dimensional parameter $\lambda$ and reverting to dimensional length scales, the above condition can be expressed as $\sigma / L_{\mathrm{B}}>4 \pi v_{0}$. Using the values $\mathrm{e}=1.60 \times 10^{-19} \mathrm{C}, \varepsilon_{0}=8.85 \times 10^{-12} \mathrm{CV}^{-1} \mathrm{~m}^{-1}$, $\varepsilon_{r}=78.3$, and $k_{\mathrm{B}}=1.38 \times 10^{-23} \mathrm{~J} \mathrm{~K}^{-1}$, the Bjerrum length can be evaluated as a function of the temperature $T$ (yielding, e.g., $L_{\mathrm{B}}=7.1 \AA$ for $T=300 \mathrm{~K}$ ) and a minimal value $\sigma_{0}$ for the mean ionic diameter can be computed. This value is reported in the the third and fourth columns of Table 1 for $T=300$ and $T=350 \mathrm{~K}$ respectively. The condition $\sigma>\sigma_{0}$ is easier to fulfill when the temperature increases. This condition is also more stringent for 1:2 and 2:2 electrolytes than for 1:1 electrolytes. Interestingly, the condition $\sigma>\sigma_{0}$ shows that the mean ionic diameter cannot take extremely low values within the present physical model (in particular, the Debye-Hückel limit $\sigma \rightarrow 0$ does not yield here a convex entropy).

\begin{tabular}{|c|c|c|c|}
\hline$Z_{+}: Z_{-}$ & $v_{0}$ & \multicolumn{2}{|c|}{$\sigma_{0}(\AA)$} \\
\hline- & - & $T=300 \mathrm{~K}$ & $T=350 \mathrm{~K}$ \\
\hline $1: 1$ & $6.263 \times 10^{-3}$ & 0.560 & 0.480 \\
\hline $2: 1$ & $2.606 \times 10^{-2}$ & 2.329 & 1.996 \\
\hline $2: 2$ & $2.506 \times 10^{-2}$ & 2.239 & 1.919 \\
\hline
\end{tabular}

Table 1. Threshold values for which assumption $\left(\mathrm{H}_{\gamma} 2\right)$ holds true.

\subsection{A periodic medium with charged disks}

Referring to $\S 2$, we consider a two-dimensional setting where $\Omega_{S}$ is a disk of radius $R=0.3 L_{*}$ whose center coincides with that of the elementary cell $\left[0, L_{*}\right]^{2}$. We take $L_{*} \in\{1,10\} \mathrm{nm}, T=300 \mathrm{~K}$, and $\Sigma_{S}=0.13 \mathrm{Cm}^{-2}$. With these values, the Debye length is in the range $[3.345,105.8] \AA$. We consider a $1: 1$ electrolyte. The mean ionic concentrations (in dimensional form) are specified as

$$
c_{+}^{0}=\frac{1}{|\Omega|} \int_{\partial \Omega} \frac{1}{\mathrm{e}} \Sigma_{S}+c^{\mathrm{salt}}, \quad c_{-}^{0}=c^{\mathrm{salt}},
$$


and we set the concentration of added salt to $c^{\text {salt }}=0.15 \mathrm{~mol} / \mathrm{l}$. In particular, since the disk is negatively charged, there is always an excess of counter-ions to ensure the global electro-neutrality of the system. It is readily seen that assumptions $\left(\mathrm{H}_{c^{0}}\right)$ and $\left(\mathrm{H}_{\Omega}\right)$ hold true. Moreover, the mean ionic diameter is set to $\sigma \in\{3,4,5\} \AA$, so that assumptions $\left(\mathrm{H}_{\gamma}\{1,2\}\right)$ also hold true. Figure 2 (left) depicts the activity coefficient $\log \gamma_{0}$ as a function of ionic strength for the various values of the parameter $\sigma$, whereas Figure 2 (right) depicts the hard-sphere activity coefficient $\log \gamma^{\mathrm{HS}}$ as a function of total concentration. This figure illustrates that electrostatic correlations have a more sizable effect for small values of $\sigma$, whereas the opposite effect is observed for steric exclusion effects.
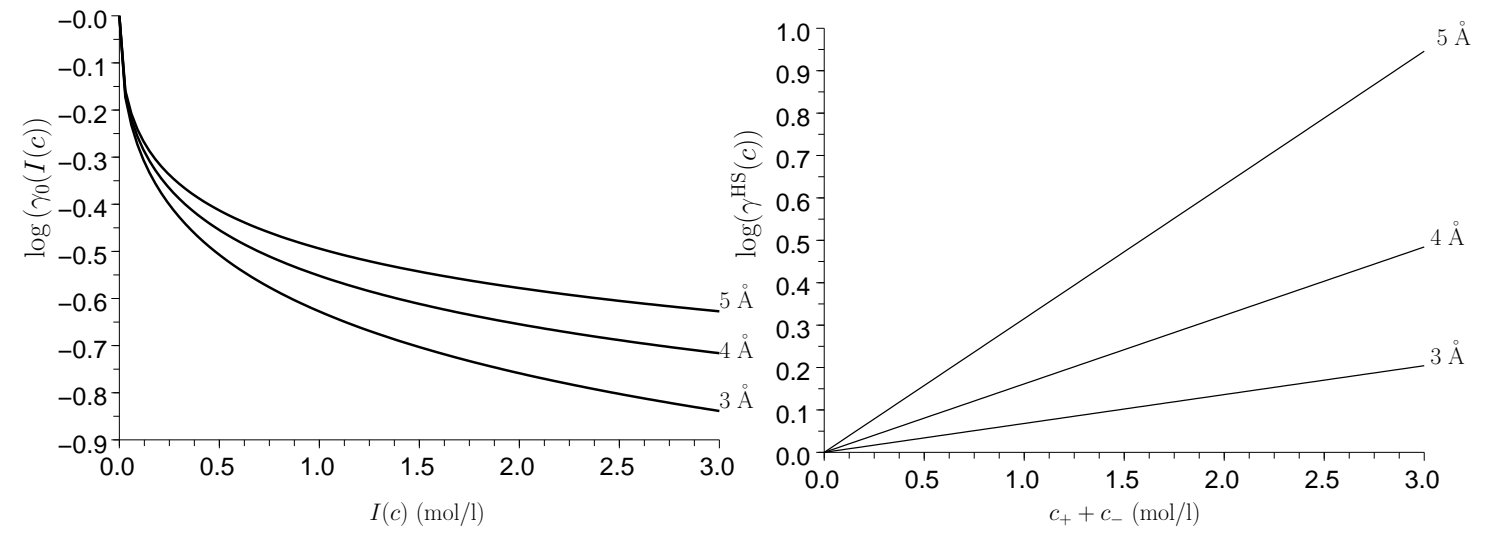

Figure 2. Left: activity coefficient $\log \gamma_{0}$ as a function of ionic strength $I(c)(\mathrm{mol} / \mathrm{l})$; Right: hard-sphere activity coefficient $\log \gamma^{\mathrm{HS}}$ as a function of total concentration $\left(c_{+}+c_{-}\right)(\mathrm{mol} / \mathrm{l})$

The saddle point of the free-energy functional is computed numerically by solving the associated Euler-Lagrange conditions approximately using conforming finite elements for space discretization (using the FreeFEM++ library [9]) in conjunction with a Newton-Raphson algorithm to solve the discrete set of coupled nonlinear equations. The constraints on the mean value of the electrostatic potential and of the ionic concentrations are conveniently handled using three additional Lagrange multipliers. More details concerning the numerical approach can be found in [11]. Figure 3 depicts iso-values of the counter-ion concentration for the two values of the reference length $L_{*}$ and for a mean ionic diameter equal to $3 \AA$ (the elementary cell is rescaled to $[0,1]^{2}$ in the figure). We observe that for large $L_{*}$, boundary layers appear near the charged walls: counter-ion concentrations exhibit a steeper gradient close to the charged surface and take almost constant values in the bulk region far from the disk.

To gain further insight, we compare the solutions obtained for the three values of the parameter $\sigma \in\{3,4,5\} \AA$ and for the two values of $L_{*} \in\{1,10\} \mathrm{nm}$. We focus on the values obtained on the horizontal line $\left[0.8 L_{*}, L_{*}\right] \times\left\{0.5 L_{*}\right\}$ joining the rightmost part of the disk to the right vertical side of the elementary cell. Figure 4 depicts the values of the activity coefficient $\log \left(\gamma_{ \pm}(c)\right)$ (note that $\log \left(\gamma_{+}(c)\right)=\log \left(\gamma_{-}(c)\right.$ ) for a symmetric electrolyte), while Figure 5 depicts those of the counter-ion concentration; 

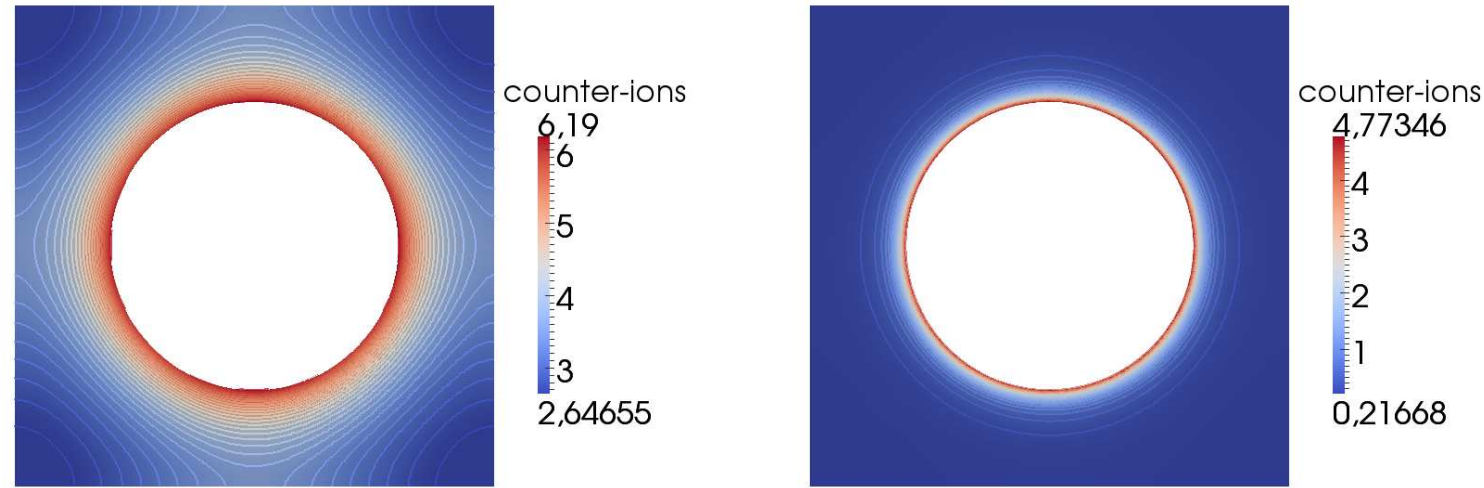

Figure 3. Counter-ion concentration (mol/l) for a 1:1 electrolyte with parameters $c^{\text {salt }}=0.15 \mathrm{~mol} / \mathrm{l}$ and $\sigma=3 \AA$. Left: $L_{*}=1 \mathrm{~nm}$; Right: $L_{*}=10 \mathrm{~nm}$.

for completeness, values obtained in the ideal Poisson-Boltzmann case $\left(\gamma_{ \pm}(c)=1\right)$ are also reported. We observe three different behaviors in Figure 4: electrostatic correlations dominate for $\sigma=3 \AA\left(\log \gamma_{ \pm}(c)>0\right)$, steric exclusion effects dominate for $\sigma=5 \AA\left(\log \gamma_{ \pm}(c)<0\right)$, or both effects play a role for $\sigma=4 \AA$. In Figure 5 , we can observe the influence of the non-ideality on the counter-ion concentration close to the charged disk, especially when comparing the concentrations to those obtained within the Poisson-Boltzmann theory. The main effect of non-ideality is to lower the counter-ion concentration close to the charged surface. Interestingly, the ideal predictions are more accurate for larger cell sizes $\left(L_{*}=10 \mathrm{~nm}\right)$.
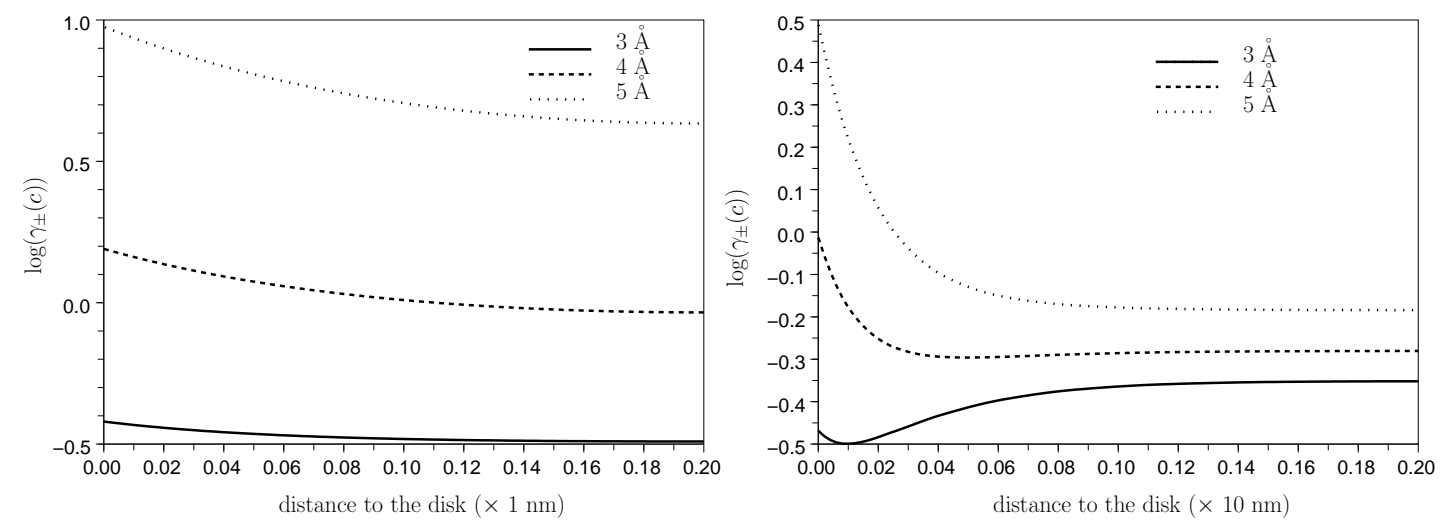

Figure 4. $\log \left(\gamma_{ \pm}(c)\right)$ for a 1:1 electrolyte with parameters $c^{\text {salt }}=0.15 \mathrm{~mol} / \mathrm{l}$ and $\sigma \in\{3,4,5\} \AA$ A. Left: $L_{*}=1 \mathrm{~nm}$; Right: $L_{*}=10 \mathrm{~nm}$.

\section{Acknowledgments}

Partial support by ANDRA (French National Agency for the Management of Nuclear Waste) is gratefully acknowledged; in particular, the second author is supported through a PhD fellowship by ANDRA. The authors are thankful to O. Bernard, 

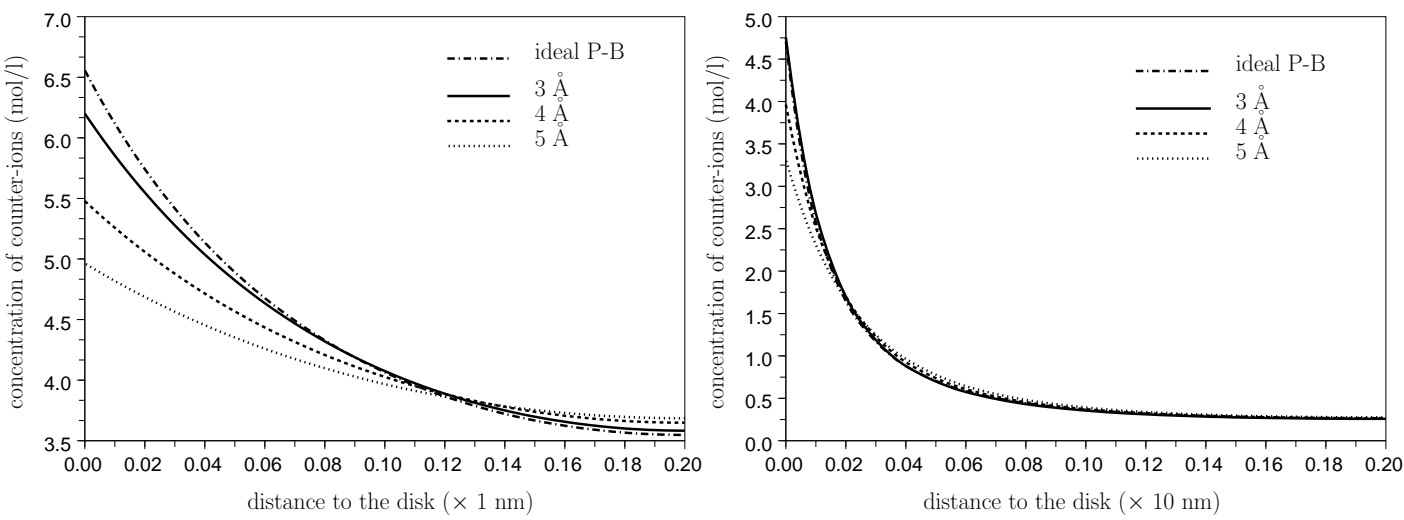

Figure 5. Counter-ion concentrations (mol/l) for a 1:1 electrolyte with parameters $c^{\text {salt }}=0.15 \mathrm{~mol} / \mathrm{l}$ and $\sigma \in\{3,4,5\} \AA$ A. Left: $L_{*}=1 \mathrm{~nm}$; Right: $L_{*}=10 \mathrm{~nm}$.

M. Jardat, B. Rotenberg, and P. Turq (PECSA, University Pierre and Marie Curie), J.F. Dufrêche (ICSM, CEA/CNRS/University of Montpellier), G. Allaire (CMAP, Ecole Polytechnique), A. Mikelić (Dep. of Mathematics, University Lyon 1), and G. Stoltz (CERMICS, University Paris-Est) for fruitful discussions.

\section{References}

[1] G. Allaire, R. Brizzi, J-F Dufrêche, A. Mikelić, and A. Piatnitski. Role of non-ideality for the ion transport in porous media: derivation of the macroscopic equations using upscaling. in preparation, 2011.

[2] G. Allaire, A. Mikelić, and A. Piatnitski. Homogenization of the linearized ionic transport equations in rigid periodic porous media. J. Math. Phys., 51(123103), 2010.

[3] L. Blum. Mean spherical model for asymmetric electrolytes. Molecular Physics, 30(5):1529-1535, 1975.

[4] I. Borukhov, D. Andelman, and H. Orland. Steric effects in electrolytes: A modified PoissonBoltzmann equation. Physical review letters, 79(3):435-438, 1997.

[5] E. A. Carlen, M. C. Carvalho, R. Esposito, J. L. Lebowitz, and R. Marra. Free energy minimizers for a two-species model with segregation and liquid-vapour transition. Nonlinearity, 16(3):1075, 2003.

[6] J. F. Dufrêche, V. Marry, N. Malíková, and P. Turq. Molecular hydrodynamics for electro-osmosis in clays: from Kubo to Smoluchowski. Journal of Molecular Liquids, 118(1-3):145-153, April 2005.

[7] I. Ekeland and R. Temam. Analyse convexe et problèmes variationnels. Dunod, 1974. Collection Études Mathématiques.

[8] J. P. Hansen and I. R. Mac Donald. Theory of simple liquids. Academic Press, 2nd edition, 1976.

[9] F. Hecht. FreeFem ++ documentation, http://www.freefem.org/ff $++/, 2011$.

[10] M. Jardat, J.-F. Dufrêche, V. Marry, B. Rotenberg, and P. Turq. Salt exclusion in charged porous media: a coarse-graining strategy in the case of montmorillonite clays. Physical Chemistry Chemical Physics, 11:2023-2033, 2009.

[11] R. Joubaud. Phd thesis. in preparation.

[12] O Kavian. Introduction à la théorie des points critiques. Springer, 1993.

[13] J.L. Lebowitz and E. Waisman. Mean spherical model integral equations for charged hard spheres. I. Method of solution. Journal of chemical physics, 56(6), 1972. 
[14] J.L. Lebowitz and E. Waisman. Mean spherical model integral equations for charged hard spheres. II. Results. Journal of chemical physics, 56(6), 1972.

[15] B. Li. Continuum electrostatics for ionic solutions with non-uniform ionic sizes. Nonlinearity, 22(4):811-833, 2009.

[16] B. Li. Minimization of electrostratic free energy and the Poisson-Boltzmann equation for molecular solvation with implicit solvent. SIAM Journal of mathematical analysis, 40(6):2536-2566, 2009.

[17] J. R. Looker. Semilinear elliptic neumann problems with rapid growth in the nonlinearity. Bulletin of the Australian Mathematical Society, 74:161-175, 2006.

[18] A. Prohl and M. Schmuck. Convergent finite element discretizations of the Navier-Stokes-NernstPlanck-Poisson system. ESAIM: Mathematical Modelling and Numerical Analysis, 44(3):531$571,2010$. 